\title{
Comparison of the Finite Volume and Discontinuous Galerkin schemes for the Double Vortex Pairing Problem using the SU2 Software Suite.
}

\author{
Kevin K. Singh ${ }^{1}$, Dimitris Drikakis ${ }^{2}$, Michael Frank ${ }^{3}$, Ioannis W. Kokkinakis ${ }^{4}$, \\ University of Strathclyde, Glasgow G1 1XJ, United Kingdom \\ Juan J. Alonso ${ }^{5}$ \\ Stanford University, Stanford, CA 94305, U.S.A \\ Thomas D. Economon ${ }^{6}$ \\ Bosch Research and Technology Center, Palo Alto, CA 94304, U.S.A \\ Edwin T. A. van der Weide ${ }^{7}$ \\ University of Twente, Enschede, 7500 AE, The Netherlands
}

\begin{abstract}
A numerical investigation of finite volume (FV) and discontinuous Galerkin (DG) finite element methods in the framework of the SU2 software is presented. The accuracy of different numerical variants is assessed with reference to the low Mach double vortex pairing flow problem, which has recently been proposed as a benchmark for studying the properties of structured and unstructured grid based methods with respect to turbulent-like vortices. The present study reveals that low-Mach corrections significantly improve the accuracy of second- and thirdorder, unstructured grid based schemes, at flow speeds in the incompressible limit. Furthermore, the $3^{\text {rd }}$-order DG method produces results similar to $11^{\text {th }}$-order accurate FV volume schemes.
\end{abstract}

\section{Introduction}

Direct numerical simulations (DNS) are used to study the intricate structures produced by complex vortexdominated flows. However, the need for fine grids and large Reynolds numbers, in conjunction with limitations in modern computing, often render the use of DNS impractical for real world applications. High-order methods can reduce the computing time, as their accuracy permits the use of a coarser grid. For problems with smooth solutions, high-order methods can provide the desired increase in accuracy with $h p$ refinement ${ }^{1}$. However, on a per-degree-offreedom basis, high-order schemes can be computationally more expensive than their low-order counterparts, 3 .

Arguably, the Finite Volume (FV) method has been the most widely used numerical method in CFD for both academic research and commercial applications. While high-order FV methods have been successfully implemented for solvers based on structured grids, their implementation in unstructured codes is complicated and often computationally costly due to the irregular shapes, arbitrary positioning and connectivity of the grid cells. Specifically, high-order methods on unstructured grids using the FV method, require a reconstruction that uses a multidimensional stencil with general cell locations, which is not trivial to implement as well as being prone to stability problems ${ }^{4}$. For this reason, most commercial CFD software packages are restricted to second order accurate

${ }^{1}$ PhD Candidate, Department of Mechanical \& Aerospace Engineering.

${ }^{2}$ Professor, Department of Mechanical \& Aerospace Engineering, AIAA Senior Life Member.

${ }^{3}$ Research Associate, Department of Mechanical \& Aerospace Engineering.

${ }^{4}$ Lecturer, Department of Mechanical \& Aerospace Engineering.

${ }^{5}$ Professor, Department of Aeronautics \& Astronautics, AIAA Associate Fellow.

${ }^{6}$ Senior Research Scientist, Multiphysics Modeling and Simulation, AIAA Senior Member.

${ }^{7}$ Assistant Professor, Department of Mechanical Engineering, AIAA Senior Member. 
methods. The Discontinuous Galerkin Finite Element Method, henceforth labeled as DG for simplicity, is a promising method that can circumvent these issues as one can increase the order of accuracy locally within each cell, potentially enabling arbitrarily high-order accurate schemes on unstructured grids ${ }^{4}$. DG formulations, because of the intrinsic computational intensity of the fundamental algorithms, can also be efficiently implemented in modern computing hardware with complex memory hierarchies and high degrees of parallelism.

In turn, an increasing number of theoretical studies compare the accuracy and computational performance of the DG and FV schemes. It has been shown that, for equivalent spatial order of accuracy, the DG scheme can produce slightly more accurate results and also converges to a solution faster than the corresponding FV schemes ${ }^{5-7}$. However, the DG scheme results in spurious oscillations close to discontinuities due to the lack of numerical dissipation $^{4,8}$ that can only be handled with appropriate shock-capturing schemes ${ }^{8,9}$. FV schemes using the weighted essentially non-oscillatory (WENO) reconstruction technique tend to be more robust than pure DG ${ }^{10,11}$, especially for flows featuring shock waves and contact discontinuities. While total variation bounded (TVB) limiters can be used within the DG framework to handle discontinuous solutions, they are still, in general, less robust than their WENO counterparts ${ }^{10}$. Other known treatments for discontinuous solutions for DG schemes is the addition of numerical dissipation in the form of artificial viscosity ${ }^{8}$, or the use of a posteriori sub-cell finite volume limiter ${ }^{9}$.

In addition to improving the accuracy of DG and FV methods in problems with discontinuous solutions, challenges also arise in the simulation of vortical flows, which requires modeling as wide a range of structure sizes as possible (i.e. maximizing the achievable dynamic range). Hence, one method of testing the suitability of the numerics is to ask the question: "What is the lowest resolution that can be used to model a basic vortical structure?" 3

This motivates us to use the double vortex pairing problem as a benchmark for the comparison of numerical schemes. The phenomenon, initially observed experimentally ${ }^{15}$, involves a mixing layer formed by bringing two streams of water, moving at different velocities together in a lucite-walled channel. The initial velocity perturbations at this interface inflate (Figure 1(a)), forming two distinct turbulent-like vortices (Figure 1(b), (c)). These two vortices interact by rolling around each other (Figure 1(d), (e)), eventually forming a single vortical structure which has approximately twice the size of the two original vortices (Figure 1(f)). This pairing process is observed to occur repeatedly, controlling the growth of the mixing layer ${ }^{3,15}$.The flow conditions (stream velocities, length-scales, Reynolds number etc.) investigated in the numerical simulations are taken from the aforementioned experiment with the aim of calculating the evolution of some of the observed large-scale structures.

The main objective of this investigation is to compare the relative performance of two different numerical schemes, the DG and FV methods, on unstructured grids for varying orders of accuracy and grid resolutions, when trying to resolve a turbulent-like vortex interaction. The results are compared to a reference solution obtained from a $11^{\text {th }}$ order accurate WENO-FV scheme on a $256 \times 256$ structured grid. The results obtained in this study were obtained using the SU2 code ${ }^{12-14}$.

The paper is organized as follows. The initial conditions for the double vortex pairing test case and a brief description of the numerical methods are described in Section II. A comparison of results between the FV and DG schemes with respect to vortex evolution and momentum thickness is presented in Section III. Finally, a series of conclusions based on the results will be presented in Section IV.

\section{Problem Description and Methodology}

\section{A. Double Vortex Pairing}

As done in previous numerical double vortex investigations ${ }^{3,24}$, the initial condition consists of two co-flowing fluids of same component and phase. The lower stream flows with velocity $u_{1}=4.06 \mathrm{~cm} / \mathrm{s}$ while the upper stream is given a velocity of $u_{2}=1.44 \mathrm{~cm} / \mathrm{s}$. The frame of reference adopted here moves with the mean stream velocity of $U$ $=2.75 \mathrm{~cm} / \mathrm{s}$ and focuses on the merger of two small vortices into one larger vortex. The final vortical structure has an approximate wavelength of $L \approx 6 \mathrm{~cm}$, which is the characteristic dimension of the computational domain.

Equal and opposite free-stream velocities are assigned to the two layers to maintain a velocity difference of $\Delta U=$ $2.62 \mathrm{~cm} / \mathrm{s}$ between the co-flowing streams. The freestream properties are listed as follows: lower stream velocity $U_{\infty}{ }^{\text {Lower }}=\Delta U / 2=1.31 \mathrm{~cm} / \mathrm{s}$; upper stream $U_{\infty}^{\text {Upper }}=-U_{\infty}^{\text {Lower }} ;$ density $\rho_{\infty}=1 \mathrm{~g} / \mathrm{cm}^{3} ;$ pressure $P_{\infty}=10.3 \mathrm{~N} / \mathrm{m}^{2}$ and kinematic viscosity $v=0.01 \mathrm{~cm}^{2} / \mathrm{s}$. The Mach number is set to $\mathrm{M}=0.2$ and is calculated based on the velocity difference between the two streams, and assuming a constant adiabatic index $\gamma=5 / 3$ of an ideal monatomic gas. The Reynolds number based on the velocity difference is $R e=\Delta U L / v=1600$.

The unperturbed streamwise velocity profile is given by equation: 


$$
u=-\frac{1}{2} \Delta U \tanh \left(\frac{y}{2 \theta_{0}}\right)
$$

where $\theta_{0}$ is the initial momentum thickness and equals $0.03 \mathrm{~cm}$. At the interface between the two streams, a stream function is used to add a divergence-free initial perturbation to both the $\mathrm{u}$ and $\mathrm{v}$ velocity components. The fluctuations are given by:

$$
u^{\prime}=-\frac{\partial \psi}{\partial y} \quad v^{\prime}=\frac{\partial \psi}{\partial x}
$$

where the stream function $\psi$ is the sum of two Kelvin-Helmholtz instability eigenmodes ${ }^{3,16}$ and is given by:

$$
\psi=A_{1}(y) \frac{v_{1}}{k_{1}} \cos \left(k_{1} x\right) \exp \left(-k_{1}|y|\right)+A_{2}(y) \frac{v_{2}}{k_{2}} \cos \left(k_{2} x\right) \exp \left(-k_{2}|y|\right)
$$

where the $k_{1}$ and $k_{2}$ are wavenumbers defined as:

$$
k_{1}=\frac{2 \pi}{L} \quad k_{2}=\frac{4 \pi}{L}
$$

and

$$
A_{i}=\frac{1-\exp \left[-2 k_{i}\left(\frac{L}{2}-|y|\right)\right]}{1-\exp \left(-k_{i} L\right)}
$$

The velocity amplitudes are given by $v_{1}=0.025 \Delta U$ and $v_{2}=0.05 \Delta U$. The boundary conditions are periodic in the $\mathrm{x}$-direction and symmetric in the y-direction. The symmetric boundary condition is employed to avoid viscous dissipation at the boundaries.

A passive scalar $(\varphi)$ is used to aid the flow visualization of the vortices and is advected with the flow according to the following equation:

$$
\frac{d \rho \varphi}{d t}+\frac{1}{V_{i}} \oint_{\partial V_{i}} \mathrm{~V}_{n} \rho \varphi d A=0
$$

where $V_{i}$ is the volume of element $i, \partial V_{i}$ is the boundary of the element $i$ and $V_{n}$ is the velocity normal to the cell area. The passive scalar is initialized with $\varphi=0$ for a negative freestream velocity (upper stream) and $\varphi=1$ for a positive freestream velocity (lower stream).

The momentum thickness $(\theta)$ is used to determine the growth rate of the mixing layer, hence enabling a quantitative comparison between the numerical schemes:

$$
\theta=\int_{y=-\infty}^{\infty} \frac{\left[u_{1}-u(y)\right]\left[u(y)-u_{2}\right]}{\left(u_{1}-u_{2}\right)^{2}} d y
$$

where $u_{1}$ and $u_{2}$ are the lower and upper stream velocities respectively. The difference in the momentum thickness produced between the reference result ( $11^{\text {th }}$ order WENO-FV) and the momentum thickness produced by the FV and DG schemes on unstructured grids within SU2, also sheds light on the relative performance of the schemes. 


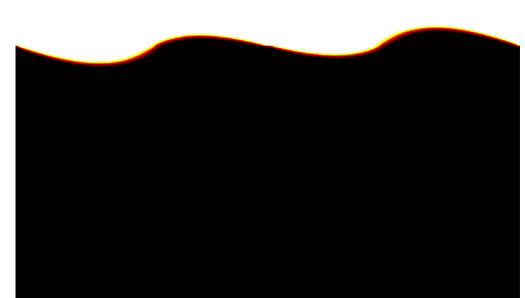

(a) $1.0 \mathrm{~s}$

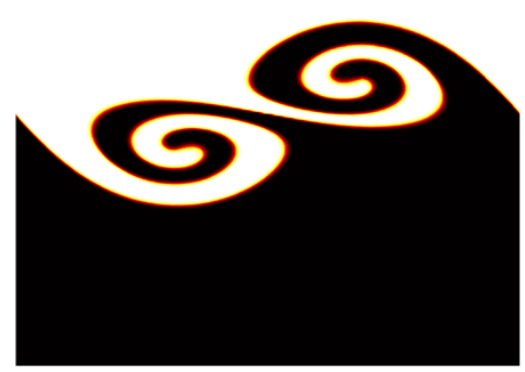

(d) $4.0 \mathrm{~s}$

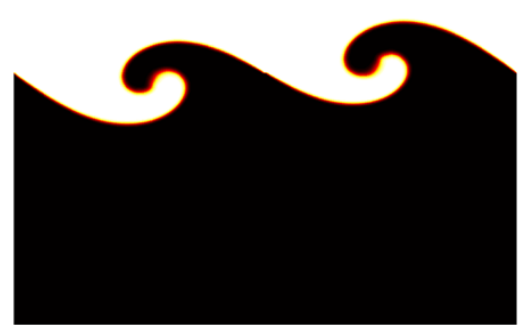

(b) $2.0 \mathrm{~s}$

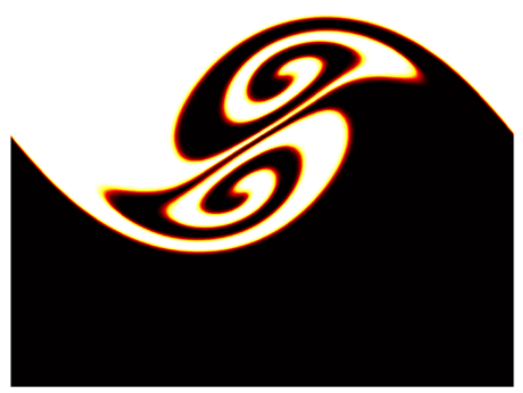

(e) $5.0 \mathrm{~s}$

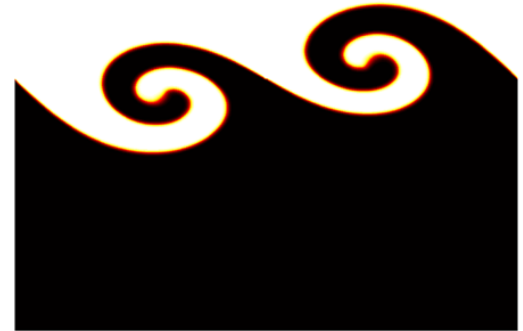

(c) $3.0 \mathrm{~s}$

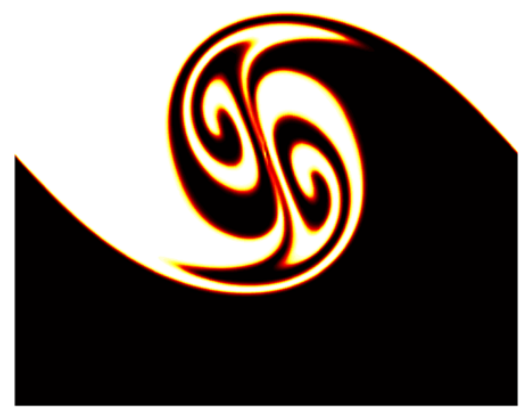

(f) $6.0 \mathrm{~s}$

Figure 1. Time evolution of the passive scalar for the double vortex pairing on a $256 \times 256$ structured grid using a $11^{\text {th }}$ order WENO-FV scheme.

\section{B. Numerical Methods}

The unstructured grid code (SU2) employs two different numerical schemes to discretize the full compressible Navier-Stokes equations, namely a vertex-centered finite volume scheme and a nodal discontinuous Galerkin Finite Element method. In all cases the inter-cell numerical fluxes are calculated by using the approximate Riemann solver HLLC, which stands for Harten, Lax, van Leer and the missing "Contact" ${ }^{17}$. The solutions are advanced in time through a $4^{\text {th }}$-order accurate Runge-Kutta method, herein referred to as RK4. It should be noted that all compressible unstructured grid framework results were obtained on a domain using triangular elements as it was previously observed that the compressible unstructured grid finite volume framework produced the best results on triangular meshes $^{3}$.

The basic implementation of the nodal discontinuous Galerkin scheme implemented within SU2 is outlined within this subsection. The DG framework uses nodal shape functions with equidistant interpolation points ${ }^{4,8,23}$. Solutions at the cell faces are discontinuous and hence handled by solving the approximate Riemann solver HLLC. Integrals are computed through use of an appropriate order of quadrature rule to avoid aliasing errors.

Within the vertex-centered finite volume scheme, a standard edge-based structure is used on a dual grid where the control volumes are constructed by using a median-dual vertex-based scheme ${ }^{12-14}$. Reconstruction of the variables to the cell faces are handled through use of the Monotone Upwind Scheme for Conservations Laws (MUSCL). The $2^{\text {nd }}$-order MUSCL (M2) scheme employs the slope limiter of Venkatakrishnan ${ }^{18}$. To obtain $3^{\text {rd }}$ order accuracy in space, the total variation diminishing (TVD) limiter from Drikakis \& Zoltak $^{19}$ was employed. The increase in accuracy from $2^{\text {nd }}$ to $3^{\text {rd }}$ order comes with virtually no increase in computational time. From here on, the $3^{\text {rd }}$ order MUSCL scheme will be referred to as M3. Convective and viscous fluxes are evaluated at the midpoint of an edge $12-14$

The reference solution was produced by using the block-structured code CNS3D that solves the full compressible Navier-Stokes equations through use of a Godunov-type finite volume solver, which has been used successfully in the past to simulate the double vortex test case ${ }^{3}$. Reconstruction of the solution to the cell faces is handled through a 
WENO reconstruction technique, which employs one-dimensional stencils and weighted polynomials to obtain highorder accuracy ${ }^{20}$. The WENO implementation is a modified version of the original scheme and contains the relative smoothness limiter of Taylor et al. ${ }^{23}$.

The finite volume schemes from both the structured and unstructured grid frameworks have also been augmented with a Low Mach Correction (LMC) which employs an additional stage in the reconstruction step to ensure uniform dissipation of the kinetic energy as the Mach number tends to zero ${ }^{22}$.

\section{Results}

The computational results from the different numerical frameworks and grid resolutions employed are presented in this section. Results from the unstructured and structured grid finite volume frameworks are presented in Section III.A. A comparison between FV and DG schemes within the SU2 framework is presented in Section III.B. Finally, the total variation bounded (TVB) properties of DG and FV are commented on in Section III.C.

\section{A. Finite Volume Solver}

\section{Vortex Evolution}

Since no analytical solution exists for the double vortex pairing case, we have chosen the results obtained from the $11^{\text {th }}$-order WENO scheme on a grid-converged resolution $(256 \times 256)$ (see Fig. 2) as the reference solution. To further test the properties of the numerical schemes, we also run the simulation with a very low Mach number $(\mathrm{M}=0.02)$ which sheds light on the schemes' resilience to dissipation due to a very low Mach number. Iso-lines for the all passive scalar plots where chosen as $0.25,0.5$ and 0.75 .

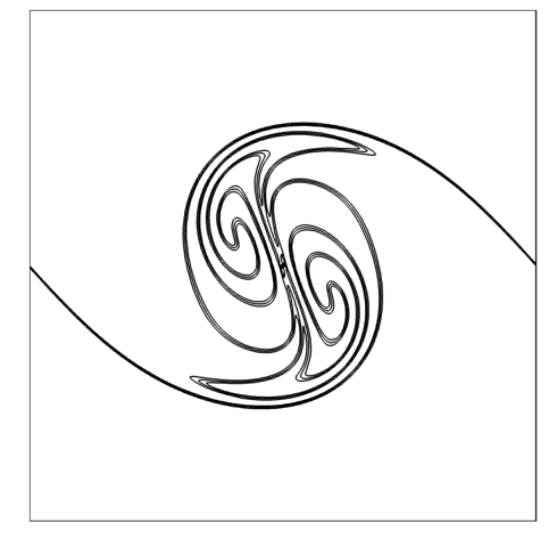

Figure 2: Passive Scalar iso-lines for the passive scalar at $t=6.0 \mathrm{~s}$ and $M=0.2$, from a $11^{\text {th }}$ order WENO scheme on a 256x256 grid with quadrilateral elements using the block-structured code CNS3D.

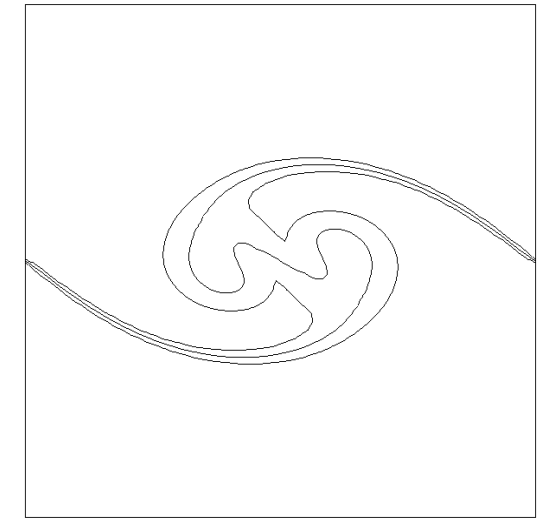

(a) No Low Mach Correction

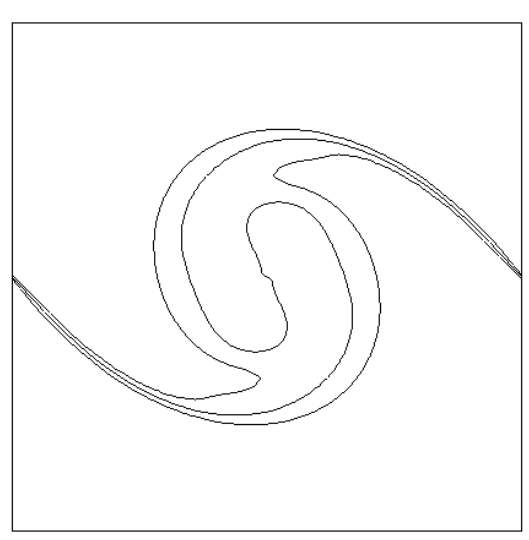

(b) Low Mach Correction

Figure 3: Passive Scalar iso-lines of the final vortex structure produced at time $t=6.0 \mathrm{~s}$ from the M2 scheme in SU2 on a 64x64 grid and Mach number $M=0.2$. 


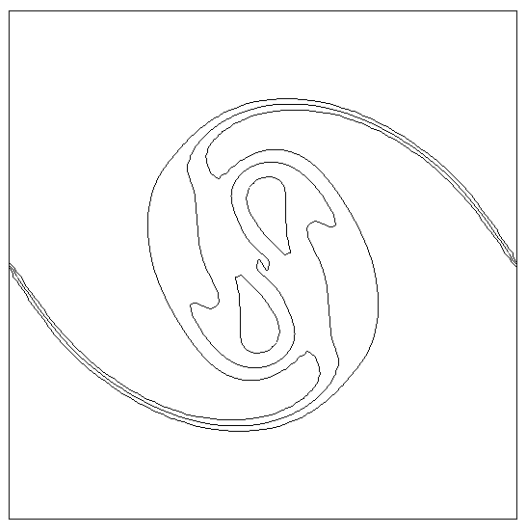

(a) No Low Mach Correction

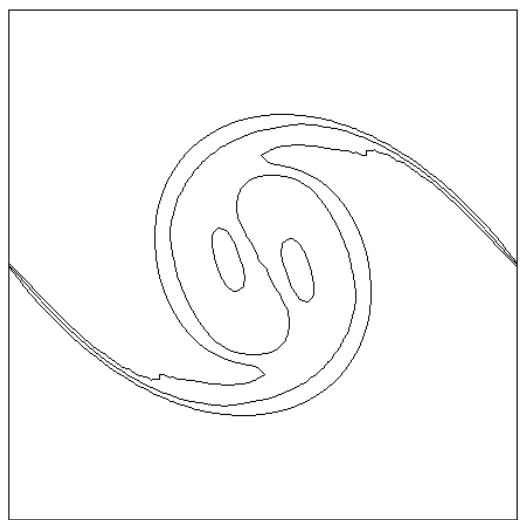

(b) Low Mach Correction

(c)

Figure 4: Passive Scalar iso-lines of the final vortex structure produced at time $t=6.0 \mathrm{~s}$ from the M3 scheme in SU2 on a 64x64 grid with Mach number $M=0.2$.

We observe that for the coarsest grid resolution, the vortical structures produced are affected by the numerical scheme employed. When no LMC is used, the dissipative nature of the M2 scheme compresses the overall shape of the paired vortex into a rounder structure (Fig 3(a)). Additionally, the vortex is rotated at a greater angle than that of the reference solution. Increasing the order of accuracy has the anticipated effect of capturing the elongated shape of the two vortices and resolving some of the finer internal structures (Fig 4(a)). With the addition of LMC, the M2 captures the vortex structures (Fig 3(b)) more accurately, which implies that the numerical schemes at this grid resolution are sensitive to low Mach number dissipation. A similar effect is observed for the FV-M3 scheme whereby the addition of LMC produces a rounder vortical structure (Fig 4(b)). We also found that the fluid structures nearer the "legs" and away from the main body of the interaction are defined in greater detail with LMC.

For reference, the solution from the block-structured code (CNS3D) exhibits similar patterns when using LMC. The correction enables a more accurate overall vortex structure and rotation angle to be calculated (Figure 5(a), 5(b)). When the low Mach dissipation is removed, the final structure produced is rounder with a more correct- with respect to the reference solution - final rotation angle. As found with the SU2 code, the "tails" of the vortices are also defined with greater detail when using LMC.

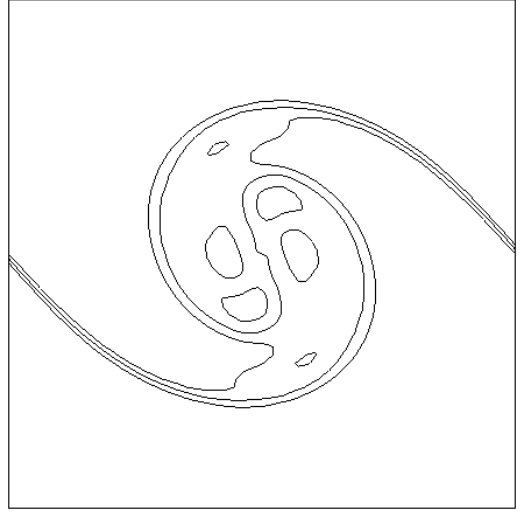

(a) No Low Mach Correction

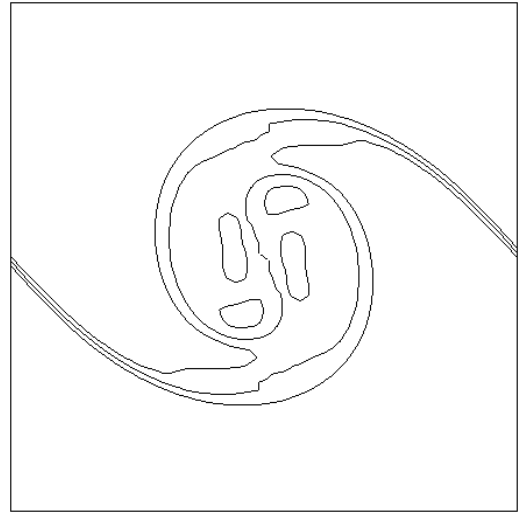

(b) Low Mach Correction

Figure 5: Passive Scalar iso-lines of the final vortex structure produced at time $t=6.0 \mathrm{~s}$ from the CNS3D-FV-M3 scheme on a $64 \times 64$ grid with Mach number $M=0.2$.

When decreasing the Mach number from $\mathrm{M}=0.2$ to $\mathrm{M}=0.02$, without $\mathrm{LMC}$, both numerical schemes (M2 and M3) are unable to resolve the roll up of the two vortices. As the Mach number is a magnitude lower than before, the dissipative effect associated with low Mach number is greater, resulting in the numerical schemes being unable to 
accurately resolve the vortex-vortex interaction (Fig 6(a)). With the application of LMC, both M2 and M3 schemes are able to capture the vortex roll up (Figure 6(b), 7(a)). Conversely, the final solution produced by the blockstructured code with LMC is similar to the results produced with the same code with a Mach number of M=0.2 (Fig $7(b))$.

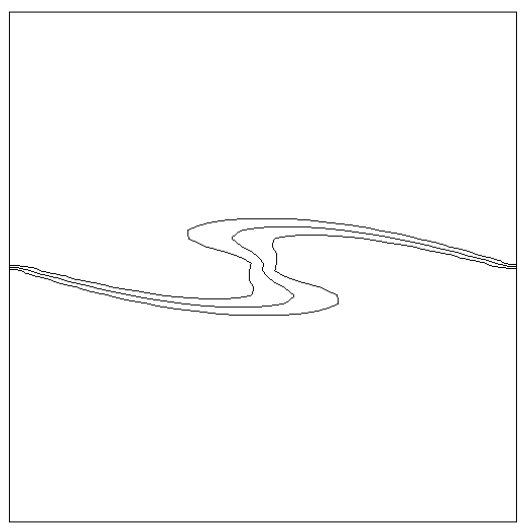

(a) No Low Mach Correction

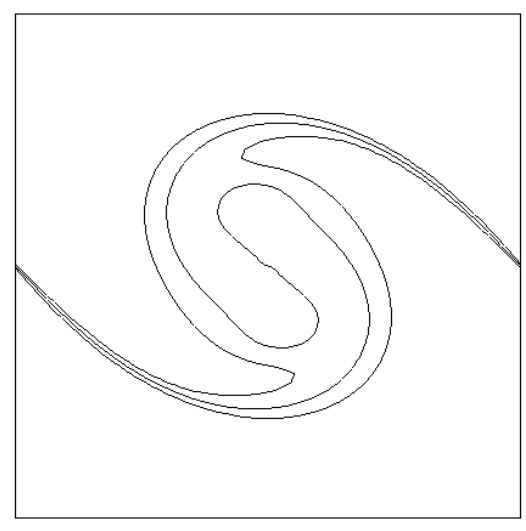

(b) Low Mach Correction

Figure 6: Passive Scalar iso-lines of the final vortex structure produced at time $t=60.0 \mathrm{~s}$ for various $\mathrm{FV}$ schemes on a 64x64 grid with Mach number $M=0.02$.

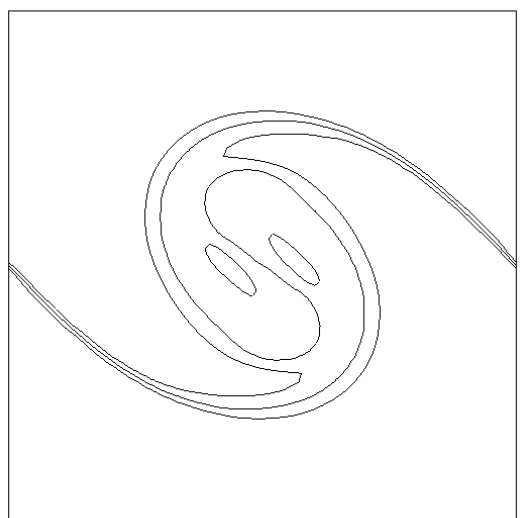

(a) SU2-M3 with Low Mach Correction

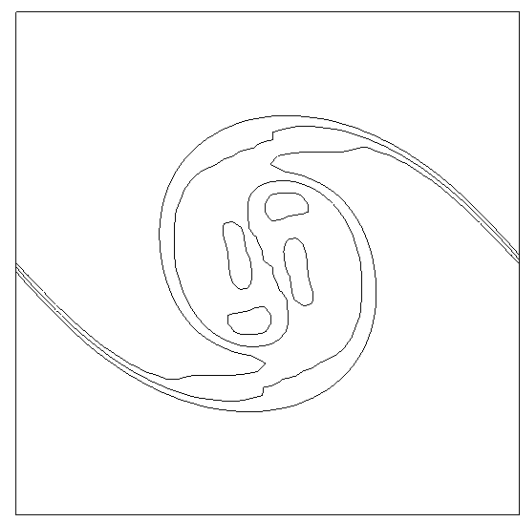

(b) CNS3D-M3 with Low Mach Correction

Figure 7: Passive Scalar iso-lines of the final vortex structure produced at time $t=60.0 s$ for various FV schemes on a 64x64 grid with Mach number $M=0.02$ and Low Mach Correction applied.

As we refine the grid resolution to a $256 \times 256$ configuration, both the M2 and M3 results improve significantly (Fig 8(a), 9(a)). However, they still do not converge to our reference solution seen in Figure 2. LMC has a lesser effect on M2 than M3; (Fig 8(a), 8(b)) and (Fig 9(a), 9(b)). The vortex evolution pattern produced from the M3 scheme with LMC compares more favourably to the reference solution than the M2 scheme. 


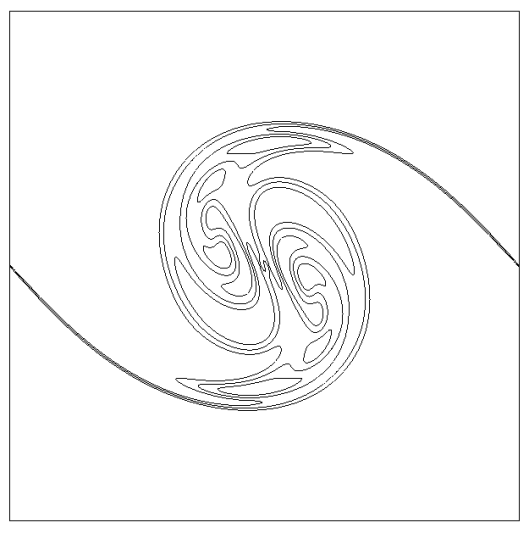

(a) No Low Mach Correction

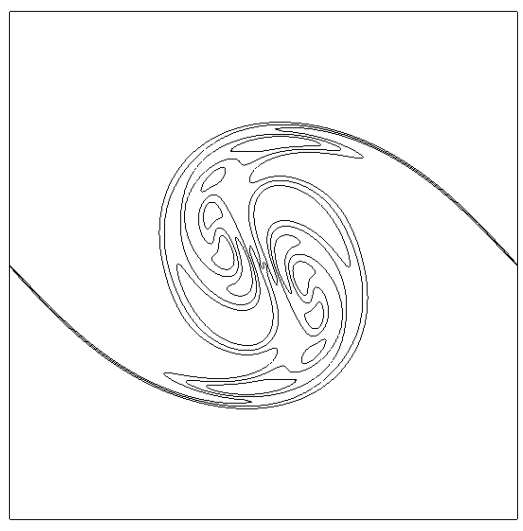

(b) Low Mach Correction

Figure 8: Passive Scalar iso-lines of the final vortex structure produced at time $t=6.0 \mathrm{~s}$ from the M2 scheme in SU2 on a 256x256 grid for Mach number $M=0.2$.

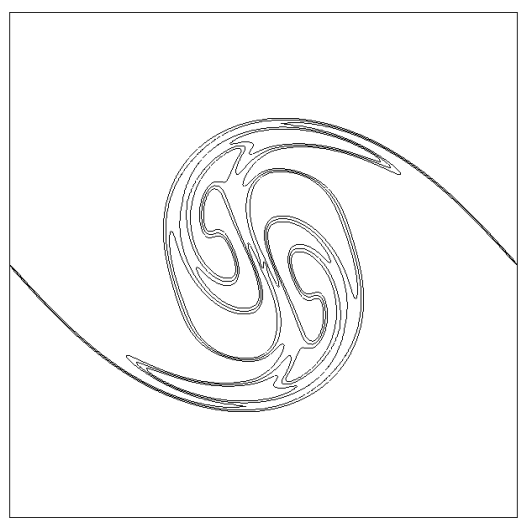

(a) No Low Mach Correction

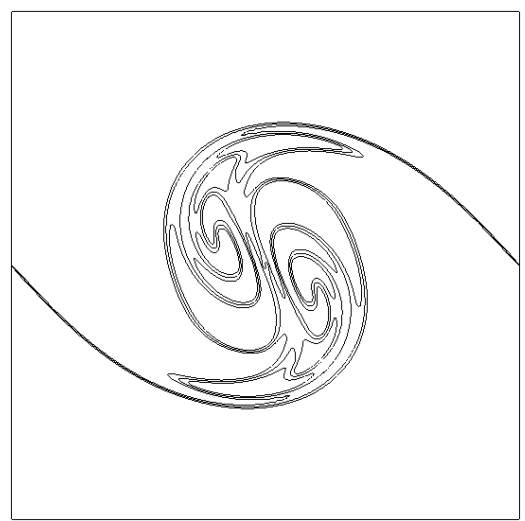

(b) Low Mach Correction

Figure 9: Passive Scalar iso-lines of the final vortex structure produced at time $t=6.0 \mathrm{~s}$ from the M3 in SU2 scheme on a 256x256 grid for a Mach number 0.2 .

\section{Momentum Thickness}

Momentum thickness growth from the initial shear layer is a distinctive characteristic of mixing in the shear layer $^{3}$, hence examination of the various numerical schemes ability to resolve this growth will enable a quantitative comparison on the relative accuracy of the schemes. We will use the temporal growth of momentum thickness observed from the reference solution $\left(11^{\text {th }}\right.$ order WENO) to indicate how accurately the numerical schemes in question resolve mixing in the shear layer. It should be noted that for the $\mathrm{M}=0.02$ case, we have normalized time with respect to the $\mathrm{M}=0.2$ case to enable a direct comparison.

For a Mach number of $\mathrm{M}=0.2$ on the coarsest grid resolution of $64 \mathrm{x} 64$, we found that neither the $-\mathrm{M} 2$ or $-\mathrm{M} 3$ were able to provide a momentum thickness profile which closely resembled the reference solution (Fig 10(a)). One observation is that the momentum thickness produced by the M3 scheme at the end of simulation at $t=6.0 \mathrm{~s}$ is overestimated and not monotone coverging. Between times $t=1.0 \mathrm{~s}$ and $\mathrm{t}=3.0 \mathrm{~s}$, we should ideally observe a non-linear mixing layer behavior, however M3 does not resolve this correctly. The application of LMC provides an improvement in the schemes' ability to accurately match the temporal growth of momentum thickness in comparison with the reference solution.

However, at late time the momentum thickness obtained from M2 and M3 are not monotonically converged (Fig 10(b)). Conversely, the higher order accuracy of the $11^{\text {th }}$ order WENO scheme shows increased resilience to low Mach number dissipation by producing a momentum thickness on a $64 \times 64$ grid, which closely matches the reference solution on a $256 \times 256$ grid. 


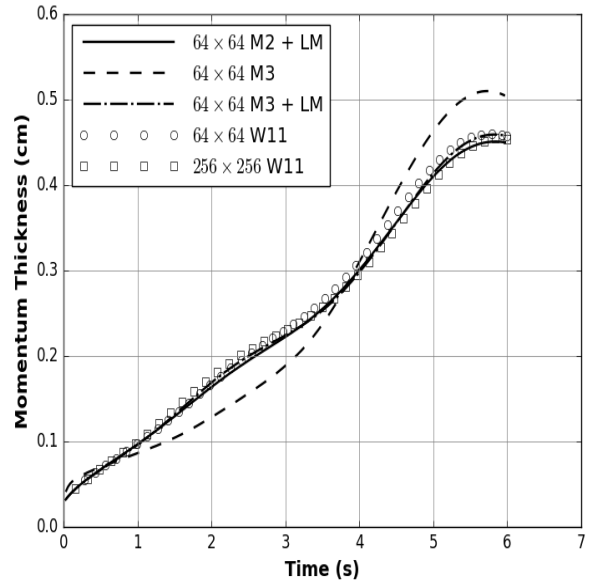

(a) $\mathrm{M}=0.2$

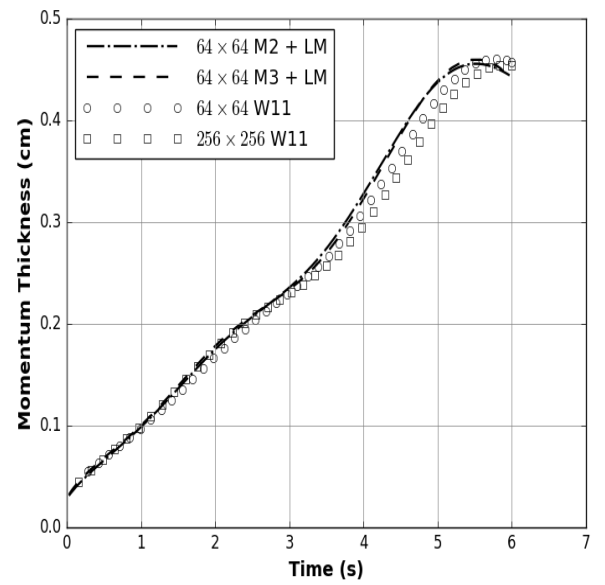

(b) $\mathrm{M}=0.02$

Figure 10: Momentum thickness evolution in time for the various schemes and Mach numbers on the 64x64 grid using SU2-FV.

\section{B. Comparison of Finite Volume and Discontinuous Galerkin Schemes}

1. Vortex Evolution.

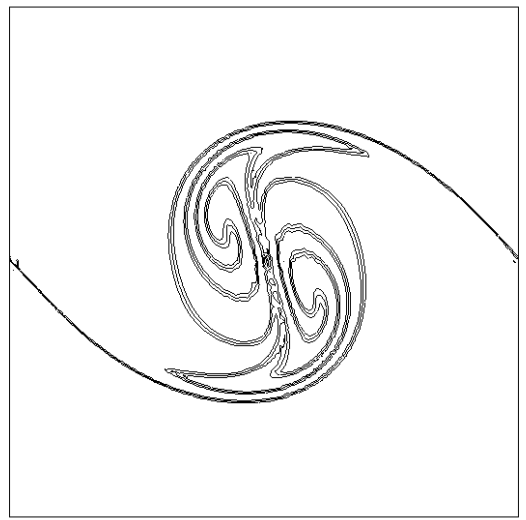

(a) $64 \times 64$

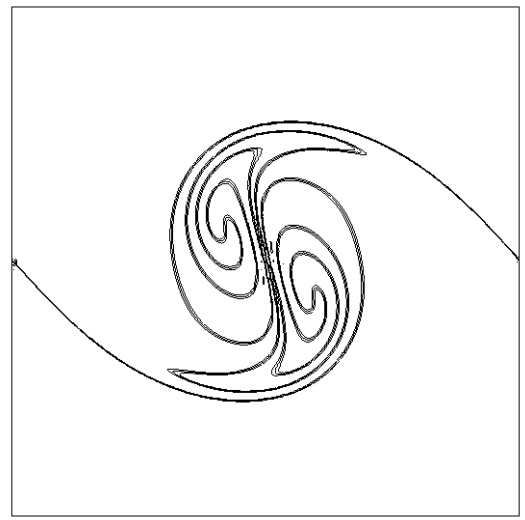

(b) $128 \times 128$

Figure 11: Passive Scalar iso-lines of the final vortex structure produced at time $t=6.0 \mathrm{~s}$ from the $3^{\text {rd }}$ order DG scheme on a for Mach number $M=0.2$ on various grid resolutions.

On a $64 \times 64$ grid, the $3^{\text {rd }}$ order DG scheme calculates a vortex with a much sharper interface and with almost identical inner structures, overall shape and orientation angle as the results obtained from the high-resolution $11^{\text {th }}$ order WENO scheme (Fig 11(a), 11(b)). Decreasing the Mach number from $M=0.2$ to $M=0.02$ (Figure 12(a), 12(b)) shows that the DG scheme, is resilient to low Mach dissipation as the vortex evolution pattern resolves a solution which very closely resembles the $\mathrm{M}=0.2$ counterparts. 


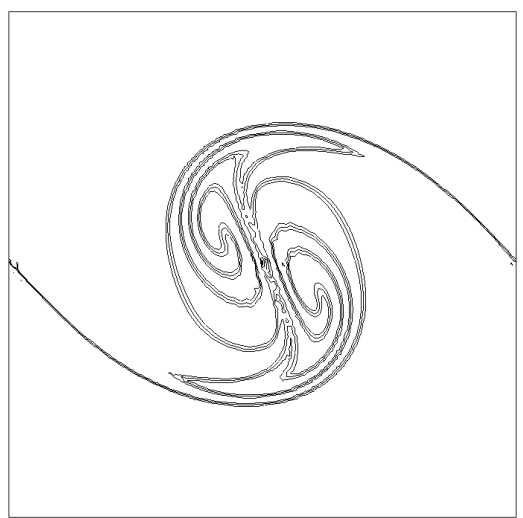

(a) $64 \times 64$

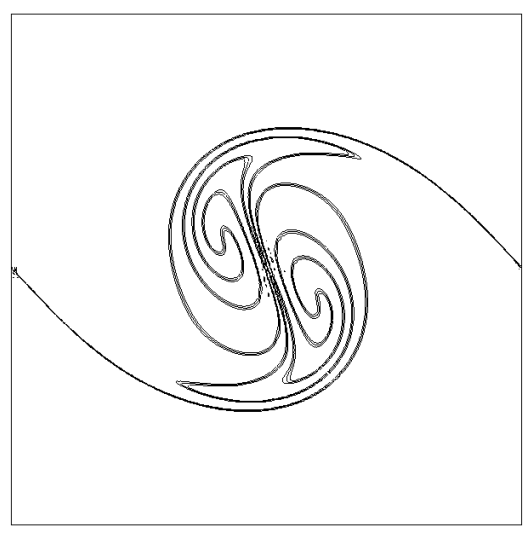

(b) $128 \times 128$

Figure 12: Passive Scalar iso-lines of the final vortex structure produced at time $t=60.0 \mathrm{~s}$ from the $3^{\text {rd }}$ order $D G$ scheme on a 128x128 grid for Mach number $M=0.02$.

\section{Momentum Thickness.}

Accurately capturing the non-linear mixing layer between $t=1.0 \mathrm{~s}$ and $\mathrm{t}=3.0 \mathrm{~s}$, as well as producing a final correct monotone convergent momentum thickness, indicates the accuracy with which the numerical scheme has captured the interaction ${ }^{3}$. The $3^{\text {rd }}$ order DG is capable of resolving the non-linear mixing layer, but provides less growth of the momentum thickness at late time (Fig 13). The DG solution on 128x128 is similar to M2 and M3 solution on $256 \times 256$ up to about 3 seconds. After that time, DG gives a slower momentum thickness growth than M2 and M3 but at the end of the simulation all schemes converge to the same value.

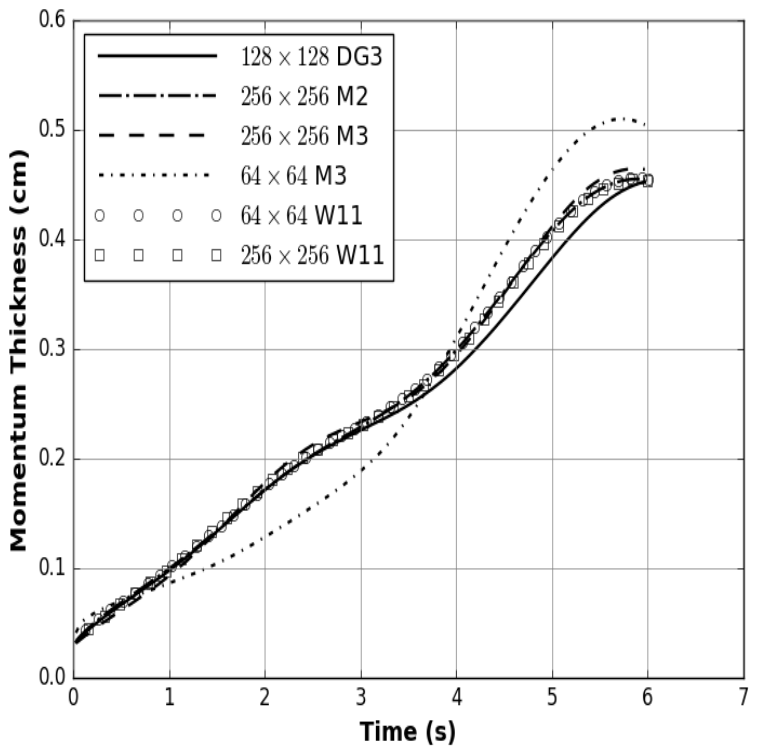

Figure 13: Momentum thickness evolution in time for the DG method on the 128x128 grid, FV (M2 \& M3) on a 64x64 and a 256x256 grid and WENO FV on a 256x256 grid at Mach number $M=0.2$.

\section{Total Variation Bounded (TVB) Property}

In this subsection we comment on the TVB property of the above schemes. It should be noted that the lower bound for the passive scalar is zero and the upper bound takes a value of one. Any values observed from simulations, which lie outside these bounds is assigned as an over/under shoot accordingly. For the following TVB plots, cells which contain a value above 1.01 are coloured black, cells which contain a value less than -0.01 are coloured in blue. Fig. 14 shows that DG produces both over/under shoots inside and around the vortex as well as leads to some oscillations throughout the flow field. On the other hand, for the same grid resolution, a $11^{\text {th }}$ order 
WENO scheme contains fewer cells with under and over shoots. The DG with lowered Mach number produces an almost identical portrait as the $\mathrm{M}=0.2$ case (Fig 15(a)). Conversely, For the lower Mach number case of $\mathrm{M}=0.02$, we observe that the lowered Mach number results in a smaller region with over and undershoots for the $11^{\text {th }}$ order WENO scheme (Fig 15(b)).

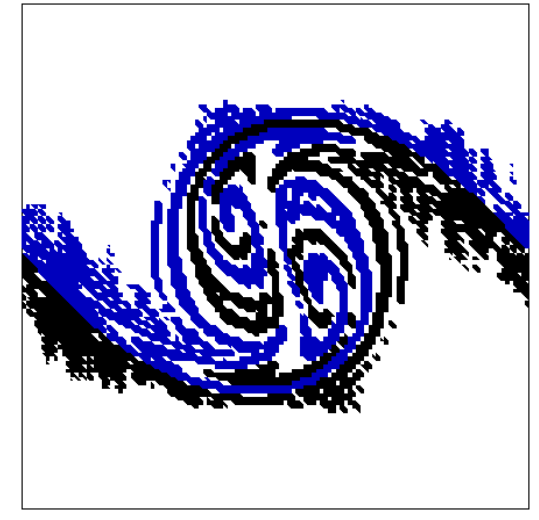

(a) $3^{\text {rd }}$ order DG

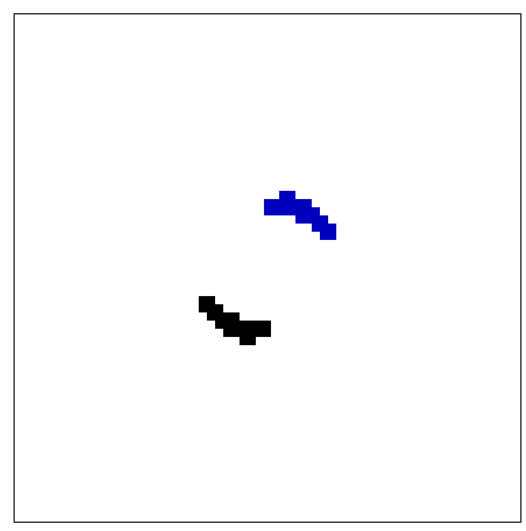

(b) $11^{\text {th }}$ order WENO-FV

Figure 14: Passive Scalar iso-lines of the final vortex structure produced at time $t=6.0$ s from the $3^{\text {rd }}$ order DG scheme on and $\mathrm{a}^{\text {th }}$ order WENO scheme on a $64 \times 64$ grid with Mach number $M=0.2$. Black coloured cells indicate an overshoot, blue coloured cells indicate an undershoot.

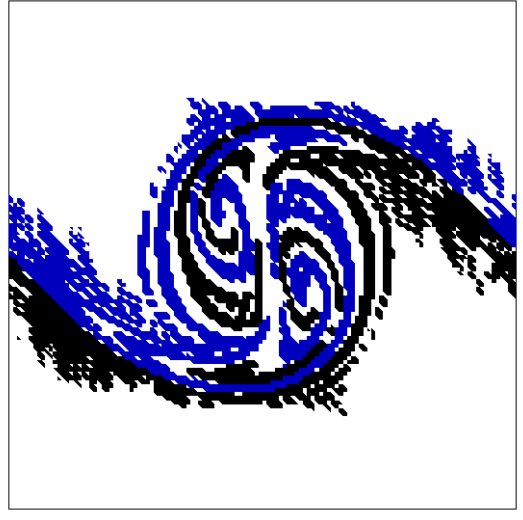

(a) $3^{\text {rd }}$ order DG

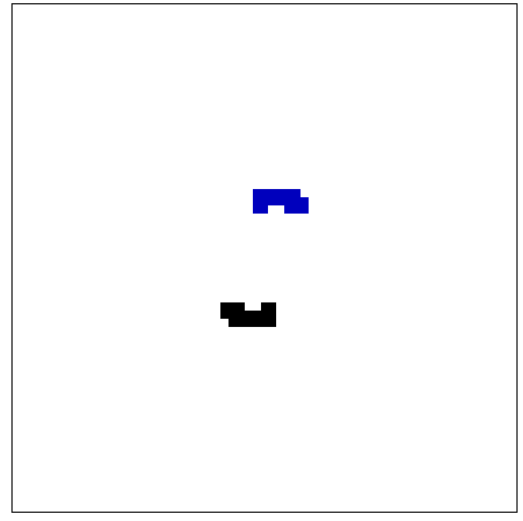

(b) $11^{\text {th }}$ order WENO-FV

Figure 15: Passive Scalar iso-lines of the final vortex structure produced at time $t=60.0 . s$ from the $3^{\text {rd }}$ order DG scheme and $a 11^{\text {th }}$ order WENO scheme on a $64 \times 64$ grid resolution at Mach number $M=0.02$. Black coloured lines indicate an overshoot, blue coloured lines indicate an undershoot.

The manner in which the high-order polynomial is constructed for a WENO scheme differs fundamentally from the polynomial construction in DG. The "weighted essentially-non-scillatory" part derives from the weno weights used in the polynomial construction process, which are used to bound the solution, thus resulting in fewer over/under shoots, as shown in Figs 14(b), 15(b).

Increasing the grid resolution shows the overshoots are now more concentrated near the discontinuities, albeit still occur in some parts of the flow field away from the discontinuities (Fig 16(a)). Decreasing the Mach number to $M=0.02$ appears to lessen the oscillatory behavior (Fig 17(a)). On the other hand, increasing the grid resolution for the $11^{\text {th }}$ order WENO scheme increases the regions of the flow which contain over/under shoots (Fig 16(b)). Finally, the decreased Mach number results in the WENO $11^{\text {th }}$ order scheme containing fewer cells with over and under shoots (Fig 17(b)). 


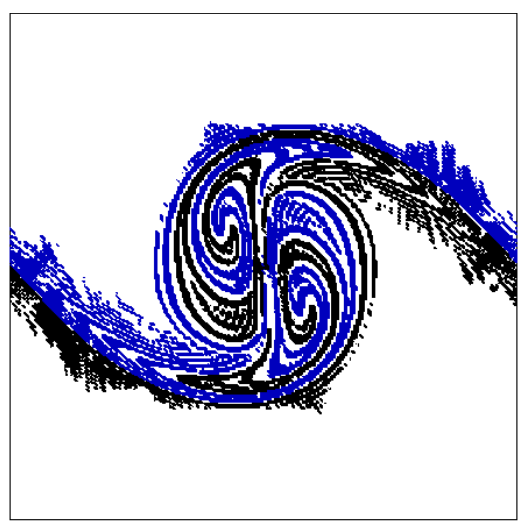

(a) $3^{\text {rd }}$ order DG

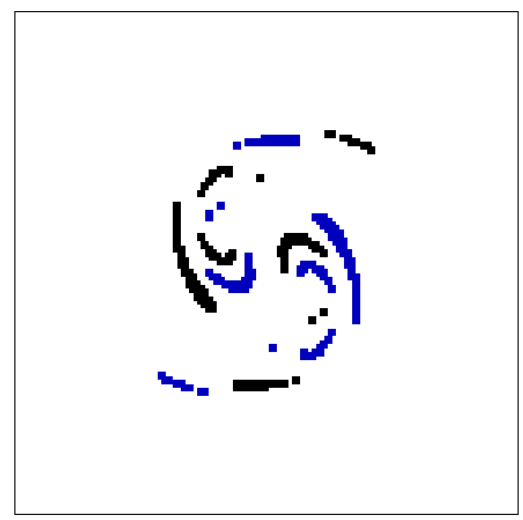

(b) $11^{\text {th }}$ WENO-FV

Figure 16: Passive Scalar iso-lines of the final vortex structure produced at time $t=6.0 \mathrm{~s}$ from a $3^{\text {rd }}$ order DG scheme and a $11^{\text {th }}$ order WENO scheme on a $128 \times 128$ grid with Mach number $M=0.2$. Black coloured cells indicate an overshoot, blue coloured cells indicate an undershoot.

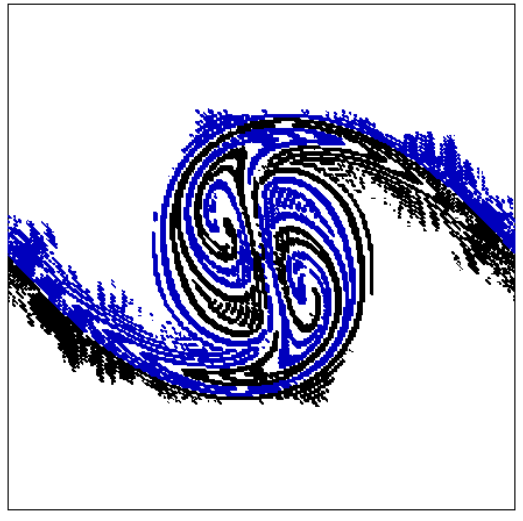

(a) $3^{\text {rd }}$ order DG

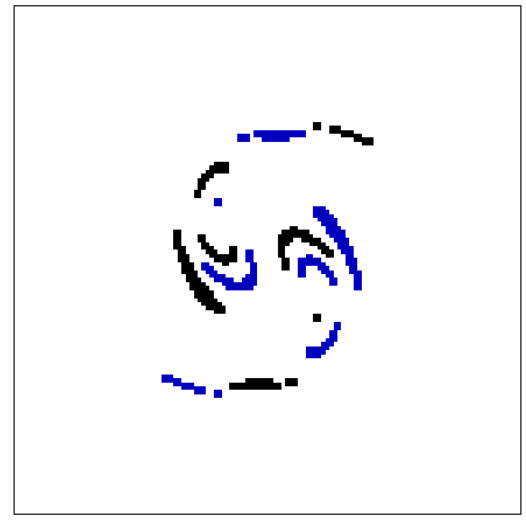

(b) $11^{\text {th }}$ order WENO-FV

Figure 17: Passive Scalar iso-lines of the final vortex structure produced at time $t=60.0 s$ from a $3^{\text {rd }}$ order DG scheme and a $11^{\text {th }}$ order WENO scheme on a 128x128 grid with Mach number $M=0.02$. Black coloured lines indicate an overshoot, blue coloured cells indicate an undershoot.

\section{Conclusions}

The main aim of this investigation was to compare the performance of $2^{\text {nd }}$ and $3^{\text {rd }}$ order accurate FV schemes to a $3^{\text {rd }}$ order accurate DG scheme on unstructured grids using the double vortex pairing problem as a test case. We would like to briefly mention that a comparison of schemes based on a grid size does not account for the additional degrees of freedom which exist within the DG scheme. Hence, a comparison evaluating the performance per degree of freedom will form part of a future study. The main findings of this numerical investigation are outlined below.

1) The addition of LMC greatly improved the results produced from the -FV solver for M2 and M3 schemes when investigating the vortex evolution pattern at the end of simulation and the temporal evolution of the momentum thickness.

2) $A 3^{\text {rd }}$ order accurate DG scheme is capable of resolving the finer features of the double vortex interaction on the coarsest grid resolution of $64 \times 64$. Increasing the grid resolution further from $64 \times 64$ to $128 \times 128$ results in the DG scheme producing an even sharper vortex pattern. The results from the vortex evolution study indicate that the DG 
schemes capture the final vortical structure with a sharper resolution than the FV scheme at the same grid resolutions.

3) The momentum thickness evolution produced on the $128 \times 128$ grid from the $3^{\text {rd }}$ order DG scheme showcases the numerical method's ability to capture the non-linear mixing layer behavior between time $t=1.0 \mathrm{~s}$ and $\mathrm{t}=3.0 \mathrm{~s}$. The DG solution for the momentum thickness develops slower than FV at late times. Both DG and FV schemes predict a similar value of momentum thickness at the end of the simulation $(t=6.0 \mathrm{~s})$.

4) When investigating the non-TVB regions produced by high-order schemes, $3^{\text {rd }}$ order DG, and $11^{\text {th }}$ order WENO, it is evident that the $3^{\text {rd }}$ order DG scheme contains larger regions of the flow with over/under shoots when compared to the $11^{\text {th }}$ order WENO. This motivates the use of TVB limiters within the DG framework and this will be part of a future study.

\section{References}

${ }^{1}$ Z. Wang, K. Fidkowski, R. Abgrall, F. Bassi, D. Caraeni, A. Cary, H. Deconinck, R. Hartmann, K. Hillewaert, H. T. Huynh, N. Kroll, G. May, P. Persson, B. v. Leer and M. Visbal, "High-Order CFD Methods: Current Status and Perspective," International Journal for Numerical Methods in Fluids, vol. 72, pp. 811-845, 2013.

${ }^{2}$ H. Huynh, Z. Wang and P. Vincent, "High-order methods for computational fluid dynamics: A brief review of compact differential formulations on unstructured grids," Computers \& fluids, vol. 98, pp. 209-220, 2014.

${ }^{3}$ P. Tsoutsanis, I. Kokkinakis, L. Könözsy, D. Drikakis, R. Williams and D. Youngs, "Comparison of structured- and unstructured-grid, compressible and incompressible methods using the vortex pairing problem," Computer Methods in Applied Mechanics and Engineering, vol. 293, pp. 207-231, 2015.

${ }^{4}$ J. Hesthaven and T. Warburton, Nodal Discontinuous Galerkin Methods: Algorithms, Analysis and Applications 1st Edition., New York: Springer Science + Business Media, 2008.

${ }^{5}$ K. H. Iqbal, "Comparison of High-Order Methods on Unstructured Grids," Ph.D. Thesis, School of Engineering, Cranfield University, Cranfield, 2013.

${ }^{6}$ T. Zhou, Y. Li and C. Shu, "Numerical Comparison of WENO Finite Volume and Runge-Kutta Discontinuous Galerkin Methods," Journal of Scientific Computing, vol. 16, no. 2, pp. 145-171, June 2001.

${ }^{7}$ J. Moortgat, M. A. Amooie and M. R. Soltanian, "Implicit finite volume and discontinuous Galerkin methods for multicomponent flow in unstructured 3D fractured porous media," Advances in Water Resources, vol. 96, pp. 389-404, 2016.

${ }^{8}$ P. Persson and J.Peraire, "Sub-Cell Shock Capturing for Discontinuous Galerkin Methods," in 44th AIAA Aerospace Sciences Meeting and Exhibit., Reno, Nevada, 2006.

${ }^{9}$ M. Dumbser and R. Loubere, "A simple robust and accurate a posteriori sub-cell finite volume limiter for the discontinuous Galerkin method on unstructured meshes," Journal of Computational Physics, vol. 319, pp. 163-199, 2016.

${ }^{10}$ C. Shu, "High Order Finite Difference and Finite Volume WENO Schemes and Discontinuous Galerkin Methods for CFD," International Journal of Computational Fluid Dynamics, vol. 17, no. 2, pp. 107-118, 2003.

${ }^{11}$ J. Cheng and C. Shu, "High Order Schemes for CFD: A Review," Chinese Journal of Computational Physics, vol. 26(5), pp. 633-655, 2009.

${ }^{12}$ F. Palacios, T. D. Economon, A. Aranake, S. R. Copeland, A. K. Lonkar, T. W. Lukaczyk, D. E. Manosalvas, K. R. Naik, A. S. Padron, B. Tracey, A. Variyar and J. J. Alonso, "Stanford University Unstructured (SU2): Open-source Analysis and Design Technology for Turbulent Flows," in AIAA SciTech, 52nd Aerospace Scienced Meeting, Maryland, 2014.

${ }^{13}$ F. Palacios, M. Colonno, A. C. Aranake, A. Campos, S. R. Copeland, T. D. Economon, A. K. Lonkar, T. W. Lukacyzk, T. W. R. Taylor and J. J. Alonso, "Stanford University Unstructured (SU2): An open-source integrated computational environment for multi-physics simulation and design," in 51st AIAA Aerospace Sciences Meeting including the New Horizons Forum and Aerospace Exposition, Dallas/Ft. Worth, 2013.

${ }^{14}$ T. Economon, F. Palacios, S. Copeland, T. W. Lukaczyk and J. J. Alonso, "SU2: An Open-Source Suite for Multiphysics Simulation and Design," AIAA Journal, vol. 54, no. 3, pp. 828-846, 2016.

${ }^{15} \mathrm{C}$. Winant and F. Browand, "Vortex pairing: the mechanism of turbulent mixing-layer growth at moderate Reynolds number," Journal of Fluid Mechanics, vol. 63, pp. 237-255, 1974.

${ }^{16}$ P. Drazin and W. Reid, Hydrodynamic Stability, Cambridge University Press, 2004.

${ }^{17}$ E. F. Toro, M. Spruce and W. Speares, "Restoration of the contact surface in the HLL-Riemann solver," Shock Waves, vol. 4, no. 1, pp. 25-34, 1994. 
${ }^{18} \mathrm{~V}$. Venkatakrishnan, "On the accuracy of limiters and convergence to steady state solutions," in AIAA 31st Aerospace Sciences Meeting \& Exhibition, Reno, Nevada, 1993.

${ }^{19}$ J. Zoltak and D. Drikakis, "Hybrid Upwind Methods for simulation of unsteady shock-wave diffraction over a cylinder," Computer Methods in Applied Mechanical Engineering, vol. 162, pp. 165-185, 1998.

${ }^{20}$ X.-D. Liu, S. Osher, T. Chan, "Weighted essentially Non-oscillatory schemes," Journal of Computational Physics, vol. 115, pp. 200-212, 1994.

${ }^{21}$ E.M. Taylor, M. Wu, M.P. Martin, "Optimization of non-linear error for weighted essentially non-oscillatory methods in direct numerical simulations of compressible turbulence," Journal of Computational Physics, vol. 223, pp. 384-397, 2007.

${ }^{22}$ B. Thornber, A Mosedale, D. Drikakis, D. Youngs, R. Williams, “An improved reconstruction method for compressible flows with low Mach number features," Journal of Computational Physics, vol. 227, pp. 4873-4894, 2008.

${ }^{23}$ J. S. Hesthaven, and T. Warburton, "Nodal high-order methods on unstructured grids. I. Time-domain solution of Maxwell's equations," Journal of Computational Physics., vol. 181, No. 1, pp. 186-221, 2002.

${ }^{24}$ S.Shanmuganathan, D.L. Youngs, J. Griffond, B. Thornber, R.J.R. Williams, "Accuracy of high order density based compressible methods in low Mach vortical flows," International Journal for Numerical Methods in Fluids, vol. 74, no. 5, pp. 335-358, 2014. 